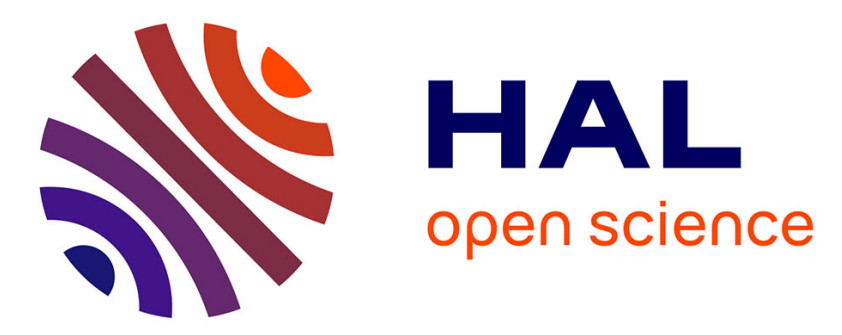

\title{
SiPM-based Underwater Wireless Optical Communication Using Pulse-Amplitude Modulation
}

Mohammad Ali A Khalighi, Hassan Akhouayri, Steve Hranilovic

\section{To cite this version:}

Mohammad Ali A Khalighi, Hassan Akhouayri, Steve Hranilovic. SiPM-based Underwater Wireless Optical Communication Using Pulse-Amplitude Modulation. IEEE Journal of Oceanic Engineering, 2020, 45 (4), pp.1611. 10.1109/JOE.2019.2923501 . hal-02421565

\section{HAL Id: hal-02421565 https://hal.science/hal-02421565}

Submitted on 9 Mar 2020

HAL is a multi-disciplinary open access archive for the deposit and dissemination of scientific research documents, whether they are published or not. The documents may come from teaching and research institutions in France or abroad, or from public or private research centers.
L'archive ouverte pluridisciplinaire HAL, est destinée au dépôt et à la diffusion de documents scientifiques de niveau recherche, publiés ou non, émanant des établissements d'enseignement et de recherche français ou étrangers, des laboratoires publics ou privés. 


\title{
SiPM-based Underwater Wireless Optical Communication Using Pulse-Amplitude Modulation
}

\author{
M. A. Khalighi ${ }^{1}$, H. Akhouayri ${ }^{1}$, S. Hranilovic ${ }^{2}$ \\ ${ }^{1}$ Aix Marseille University, CNRS, Centrale Marseille, Institut Fresnel, Marseille, France \\ ${ }^{2}$ McMaster Univeristy, Dept. Electrical \& Computer Engineering, Hamilton, ON Canada
}

\begin{abstract}
Emerging maritime applications arising from the continued growth of the marine economy have an inherent need for high data rate underwater wireless links. Within this context, underwater wireless optical communication is known as a promising technology for data transmission over shortto-medium ranges; the current available technology provides a transmission span of about $100 \mathrm{~m}$. In view of extending the transmission range, silicon photo-multipliers (SiPMs) have recently emerged as a photo-detection solution offering high receiver (Rx) sensitivity together with operational flexibility. In this paper, we introduce the use of pulse amplitude modulation (PAM) together with frequency-domain equalization (FDE) at the Rx to boost the communication rate beyond the bandwidth (BW) limitation of the optoelectronic components. For instance, for a link BW limited to $2 \mathrm{MHz}$ and 2-PAM transmission with a target BER of $10^{-4}$, the link becomes nonoperational for data rates larger than $\sim 8 \mathrm{Mbps}$ without equalization, whereas much higher data rates can be attained using FDE, e.g., 20 and 50 Mbps with maximum ranges of 28 and $10 \mathrm{~m}$, respectively, in clear waters for the SensL MicroSB-30020 SiPM and an average transmit optical power of $0.6 \mathrm{~W}$ only. Meanwhile, the nonlinear distortion of the SiPM is shown to limit the modulation order and thus the data rate in relatively short ranges. We also propose appropriate processing for PAM modulation and demodulation, given the quantum-noise-limited Rx when using an SiPM. We show that the use of non-binary PAM is undeniably advantageous for moderate data-rates (symbol rate a few MHz larger than the overall link BW) when no channel equalization is performed at the $R \mathbf{x}$. However, when employing FDE, only for very high data rates (e.g., symbol rate ten times larger than the link $\mathrm{BW}$ ), where the link frequency response becomes highly frequency selective, the non-binary PAM becomes practically interesting, outperforming 2-PAM.
\end{abstract}

Index Terms-Underwater wireless optical communications; Silicon photo-multiplier; pulse amplitude modulation; frequencydomain equalization; quantum noise.

\section{INTRODUCTION}

The on-going expansion of human activities in underwater application domains has resulted in an inherent need for reliable and high data rate wireless links. Optical wireless communications underwater offers very high data rates (more than several hundred Mbps) but over a limited range of typically several tens of meters or so. Underwater wireless optical communication (UWOC) is regarded as being costeffective, low energy consumption, high data rate, low latency, and secure [1]-[4]. Nevertheless, optical signals in water are subject to strong intensity attenuation due to light absorption and scattering. Scattering can also result in pulse broadening and inter-symbol interference (ISI) at very high data rates [5][7]. Several theoretical and experimental works have demon- strated the feasibility of this technology and several products have been commercialized (see [8] and the references therein).

Currently, one of the most important challenges for UWOC links is to considerably increase the attainable link distance. Although photo-multiplier tubes (PMTs) offer a high sensitivity thanks to their very high gain (typically $10^{6}-10^{7}$ ) and their large photo-sensitive area, they have a number of practical disadvantages including bulkiness, high cost, the requirement for high bias voltages, sensitivity to magnetic fields (due to the use of DC-to-DC converters), and vulnerability to exposition to high light intensities [8]. The use of silicon photo-multipliers (SiPMs), also called multi-pixel photon counters (MPPCs), has attracted particular attention very recently since they offer the same order of magnitude of gain as PMTs. Allowing operation over relatively long range underwater links, SiPMs have the advantages of lower cost and operating bias voltage, reduced size, weight, and power (SWaP), mechanical robustness, and insensitivity to magnetic fields, as compared with PMTs. Although currently available components suffer from limited dynamic range (DR) and relatively small active areas, due to their operational flexibility, SiPMs are likely candidates to extend the range of current UWOC systems and their use is foreseen in next generation underwater communication systems [9].

Considering the use of an SiPM at the receiver (Rx), our aim in this paper is to employ pulse amplitude modulation (PAM) in order to increase the transmission rate beyond the bandwidth (BW) limitation of the optoelectronic components used at the transmitter (Tx) and at the Rx. The motivation behind considering PAM among the other existing intensity modulation techniques is that, unlike optical orthogonal frequencydivision multiplexing (O-OFDM), considered in [10], [11], PAM exhibits a relatively low peak-to-average power ratio (PAPR), and hence, its performance is less affected by the limited DR of the Tx. Also, PAM has the advantages of lower sensitivity to synchronization errors, compared with pulse position modulation (PPM), and higher BW efficiency than pulse width modulation (PWM). Also, it is not subject to error propagation in signal demodulation, compared with digital pulse interval modulation (DPIM) [12], [13].

Although SiPMs enable long range underwater links due to their high sensitivity, we show that they also introduce some challenges at relatively shorter ranges, where the introduced nonlinear distortion (NLD) limits the modulation order and thus the data rate. Furthermore, given that the Rx is essentially quantum-noise limited when using an SiPM, we propose 
appropriate processing for PAM modulation and demodulation. Furthermore, frequency-domain equalization (FDE) is proposed at the $\mathrm{Rx}$ to alleviate the $\mathrm{Tx} / \mathrm{Rx}$ components' $\mathrm{BW}$ limitation. We show that a substantial performance improvement can be achieved through FDE at relatively high data rates.

The remainder of the paper is organized as follows. Section II presents the principles of operation of SiPMs and their limitations. In Section III, we present our main assumptions and the model description for a typical SiPM-based UWOC system. Then, PAM signal transmission and detection is considered in Section IV where we present the two cases of classical and optimal PAM signaling. Performance evaluation of the communication link is studied in Section V, where we present a set of numerical results to illustrate the limitation on the data rate and to elucidate the performance gain offered by channel equalization. We also show the interest of the optimal PAM signaling and the advantage of using non-binary PAM at high data rates. Lastly, Section VI concludes the paper.

\section{Silicon PHOTO-MULTIPLIERS FOR PHOTO-DETECTION}

\section{A. SiPM, Operation}

An SiPM is a matrix of avalanche photo-diodes (APDs), which are connected in parallel. In their "quiescent state" these APDs are biased in the Geiger mode, i.e., over their breakdown voltage $V_{\mathrm{BD}}$. As a result, the APD has a very high gain and can potentially go into avalanche when a single photon hits its photosensitive surface; thus the name of single-photon APD (SPAD) or Geiger-mode APD. These elements of an SiPM are also often called "pixels" or "micro-cells," which in fact act as "photon counters." The output of the SiPM is the sum of the photon counts of the SPADs of number $N_{\text {SPAD }}$. Obviously, the DR of the SiPM output is limited (to between 0 and $N_{\text {SPAD }}$ ).

Avalanche can also occur due to the presence of intrinsic carriers inside the active region of the APD, resulting in the socalled dark counts. On the other hand, after being triggered by an incoming photon, the resulting current pulse in a SPAD may initiate an after-pulse (mainly because of charge trapping due to silicon defects). Also, it may trigger the adjacent pixels at the same time, what is called crosstalk. These different "noise" sources affect the performance of an SiPM-based receiver $(\mathrm{Rx})$. Typical values of $V_{\mathrm{BD}}$ are around $20-30 \mathrm{~V}$ (which depend also on the temperature) and the over-voltage $V_{\mathrm{OV}}$ (the excess bias voltage beyond $V_{\mathrm{BD}}$ ) is around $2-5 \mathrm{~V}$ [14].

A SPAD is initially in it quiescent mode until a trigger occurs, due to incoming photons or a secondary effect (i.e., dark noise, after-pulse, or crosstalk), pushing the SPAD into avalanche. A quenching device should then be used to bring it back to the quiescent mode [15]. This transition is called recovery phase and the corresponding delay is called recovery time or dead-time, denoted here by $\tau_{d}$. During $\tau_{d}$, the SPAD is unable to detect a new incoming photon and is said to be saturated [16].

Quenching can be done using a passive or an active circuitry, as illustrated in Fig. 1. In the case of active quenching (AQ), see Fig. 1(a), a dedicated active circuitry senses the avalanche, and then lowers the bias voltage below $V_{\mathrm{BD}}$ during the holdoff time, and then brings it back to the quiescent value [17].

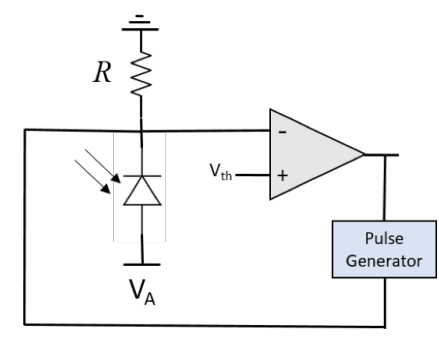

(a) AQ

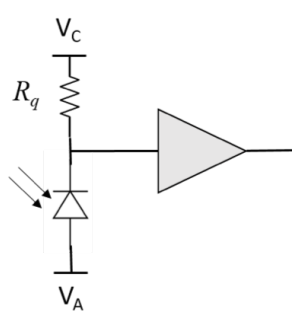

(b) PQ

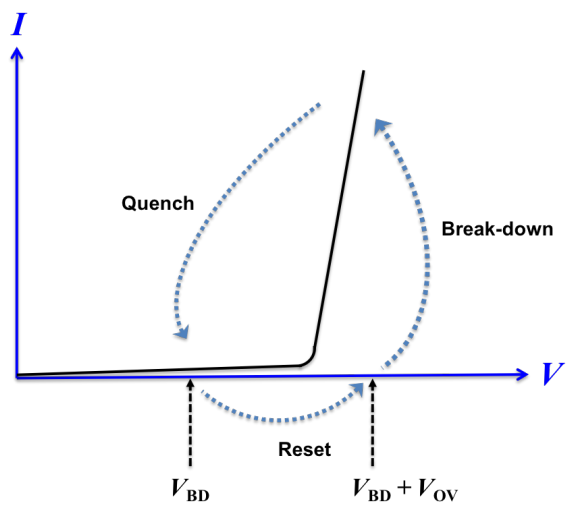

(c)

Fig. 1. (a), (b): Schematics of typical PQ and AQ SiPM circuitry [17], (c): $I-V$ charactristic of a PQ SPAD [18]

Recovery by active quenching is faster and unaffected by new incoming photons, providing the possibility of working with high count rates. However, the area required for the quenching circuit (used for every SPAD) and the control circuitry makes AQ SiPMs of very low density (i.e., very small $N_{\text {SPAD }}$; e.g., [19]), compared to passively quenched (PQ) equivalents. The simple PQ approach consists in using a quenching resistor $R_{q}$ in series with the SPAD, see Fig. 1(b). When an avalanche occurs, the voltage drop across $R_{q}$ pushes the SPAD into cutoff mode. Afterwards, the junction capacitance recharges to the bias voltage $\left(V_{\mathrm{C}}-V_{\mathrm{A}}\right.$ on the figure) during the recovery phase. Meanwhile, the new incoming photons can extend the recovery time while not being counted.

We will consider in this work the use of PQ SiPMs which are more suitable in our application as the higher $N_{\mathrm{SPAD}}$ allows working with higher intensities, i.e., shorter ranges. We will refer to the effect of the Rx saturation (i.e., signal clipping) due to dead-time limitation and the limited $N_{\text {SPAD }}$ as NLD caused on the detected signal.

In addition to $\tau_{d}$, a number of important parameters are defined for an SiPM including: the photon detection efficiency (PDE), $\Upsilon_{\mathrm{PDE}}$, i.e., the probability of detecting an incoming photon; the dark count rate (DCR), $f_{\mathrm{DCR}}$; and the probabilities of after-pulsing $P_{\mathrm{AP}}$ and crosstalk $P_{\mathrm{CT}}$. $\Upsilon_{\mathrm{PDE}}$ depends on the quantum efficiency, the over-voltage $V_{\mathrm{OV}}$, and the socalled fill-factor $\alpha_{\mathrm{FF}}$ (the ratio of the photosensitive surface to the total physical area on the chip); $f_{\mathrm{DCR}}$ increases with temperature and $V_{\mathrm{OV}}$; and $P_{\mathrm{AP}}$ and $P_{\mathrm{CT}}$ depend on $V_{\mathrm{OV}}$ and the SPAD size. 


\section{B. Accounting for Bandwidth Limitation of SiPM}

In addition to the dead-time constraint, the limited BW of SiPMs and high-power LEDs that are typically used at the $\mathrm{Tx}$, can constrain the transmission data rate. O-OFDM could be a solution to this problem [20], however, the limited DR of power LEDs and the NLD introduced by SiPMs suggests using more appropriate signaling schemes with a lower PAPR. In this work, we consider the use of simple non-return-to-zero (NRZ) PAM that can be readily employed at the Tx by simply switching on and off a set of LEDs (usually multiple LEDs are used at the $\mathrm{Tx}$ in order to increase the transmit power [9]). Note that NRZ signaling occupies a slightly larger BW (compared to root raised cosine filtering, for instance), but it has the advantage of removing the need to digital filtering at the Tx and the Rx, decreasing hence system implementation complexity and circuit power consumption.

Nevertheless, the use of (single-carrier) PAM can result in ISI at high data rates due to the BW limitation of the components, as shown in [8] for the case of on-off keying (OOK) modulation, necessitating channel equalization at the Rx. To deal with this, here we proposed the use of FDE at the $\mathrm{Rx}$ in order to boost the transmission rate far beyond the inherent BW of SiPM/LED [21], [22]. A key advantage of FDE is that it has a lower computational complexity as compared to time-domain equalization.

\section{Link Model And Main Assumptions}

We consider a typical UWOC link employing intensity modulation with direct detection. At the $\mathrm{Tx}$, we use the Lambertian model for the LED radiation pattern $P_{\mathrm{t}}$ [23]:

$$
P_{\mathrm{t}}=P_{\mathrm{Tx}} \frac{m+1}{2 \pi} \cos ^{m}(\theta), \quad \mathrm{W} / \mathrm{sr}, \quad \theta \in[0, \pi / 2],
$$

where $P_{\mathrm{Tx}}$ denotes the transmit power and $m$ is the Lambertian order which is related to the $\mathrm{Tx}$ semi-angle at half-power $\theta_{1 / 2}$ through the relationship $m=-\ln (2) / \ln \left(\cos \left(\theta_{1 / 2}\right)\right)$. We consider here an LED with $m=11$, corresponding to $\theta_{1 / 2} \approx 20^{\circ}$. We assume that the aquatic channel is frequency non-selective, i.e., we neglect pulse broadening due to multiple scattering effect, considering data rates below $100 \mathrm{Mbps}$. This is a quite reasonable assumption except in the case of highly turbid waters [5], [6], [24]. We further assume perfect TxRx alignment and negligible turbulence. The latter assumption is valid under the conditions of absence of ocean currents, negligible variations of salinity and temperature along the path [25], [26], and improbable beam blocking. To accurately estimate the channel attenuation for a given link distance $Z$, we use Monte Carlo simulations based on the oneterm Henyey-Greenstein phase scattering function model (see [1], [27] for details). For the case of a horizontal link in clear ocean waters with the typical chlorophyll concentration of $0.31 \mathrm{mg} / \mathrm{m}^{3}$ (corresponding to absorption, scattering, and attenuation coefficients of $0.069,0.08$, and $0.15 \mathrm{~m}^{-1}$ ) the channel attenuation as a function of link distance is plotted in Fig. 2.

At the $\mathrm{Rx}$, denote the signal intensity and the average photon count at the SiPM output by $P_{\mathrm{Rx}}$ and $\mu$, respectively.

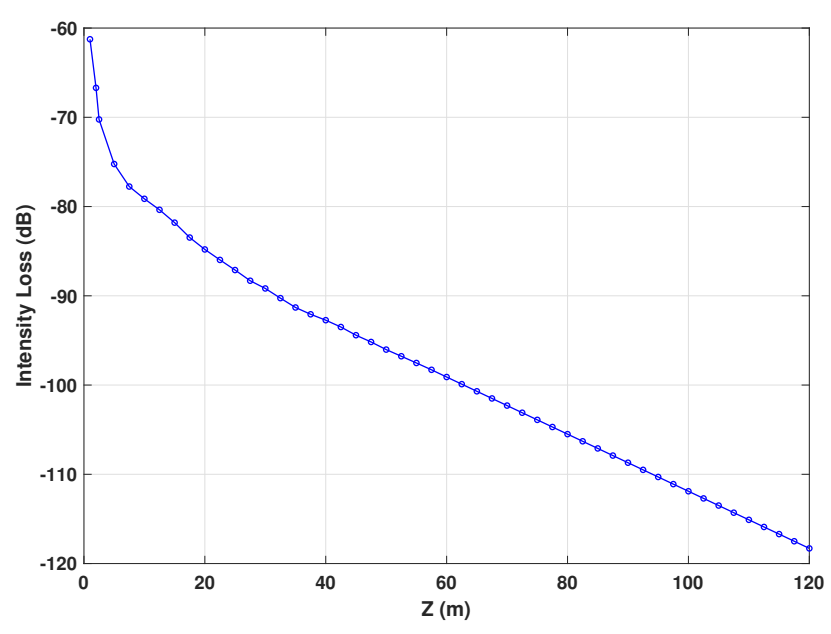

Fig. 2. Intensity loss as a function of link distance $Z$ obtained via Monte Carlo simulations, clear ocean waters with chlorophyll concentration of $0.31 \mathrm{mg} \cdot \mathrm{m}^{-3}$. Tx: LED with $m=11$; Rx: $\mathrm{FOV}=180^{\circ}$, active area $9 \mathrm{~mm}^{2}$. For $Z>80 \mathrm{~m}$, the loss values were obtained via extrapolaition.

The mean photon count can be expanded as [20]:

$$
\mu=\left(\Upsilon_{\mathrm{PDE}} P_{\mathrm{Rx}} / E_{\mathrm{ph}}+f_{\mathrm{DCR}}\right)\left(1+P_{\mathrm{AP}}+P_{\mathrm{CT}}\right) T,
$$

where $T$ is the symbol duration and $E_{\mathrm{ph}}$ the photon energy. The instantaneous photon count $\mu_{a}$ follows a Poisson distribution with parameter $\mu$ [8]. Then the average output count during $T$ for the case of a PQ SiPM is [20]:

$$
\mu_{\mathrm{PQ}}=\mu_{a} \exp \left(-\frac{\mu_{a} \tau_{\mathrm{d}}}{T N_{\mathrm{SPAD}}}\right) .
$$

Note that due to the limitation of dead-time, the probability density function (PDF) of the output photon count differs greatly from a Poisson distribution except at low photon arrival rates [28], which correspond here to practical operation ranges, as we will explain later in Section V.

In practice, it is more convenient to work with a photo-current rather than the number of detected photons, although earlier work considers a so-called photon-to-amplitude equalizer, e.g., [8], [29]. To model the output photo-current, we consider the SiPM gain $G$, which is defined as the number of generated electrons following the detection of a photon. For a given $P_{\mathrm{Rx}}$, the generated photo-current is $i_{p}=\mathcal{R} P_{\mathrm{Rx}}$, where the responsivity $\mathcal{R}$ is given by $\mathcal{R}=\Upsilon_{\mathrm{PDE}} G q / E_{\mathrm{ph}}$ and $q$ is the electron charge [18]. For the case of a PQ SiPM, the output current will be $i_{p, \mathrm{PQ}}=\mu_{\mathrm{PQ}} G q / T$.

This photo-current is converted to a voltage by the aid of a high-speed low-noise trans-impedance amplifier (TIA) with a transimpedance gain of $R$. The output is then low-pass filtered (LPF) prior to time sampling and demodulation (e.g., see Fig.3). It can be easily verified that in practice the variance of the Rx thermal noise is several orders of magnitude smaller than the signal amplitude and it can be neglected, compared to quantum noise. We further assume that our system operates in relatively deep waters so that we can neglect the effect of background illumination [30]. Thus, the dominant source of receiver noise is assumed to be quantum in nature. 


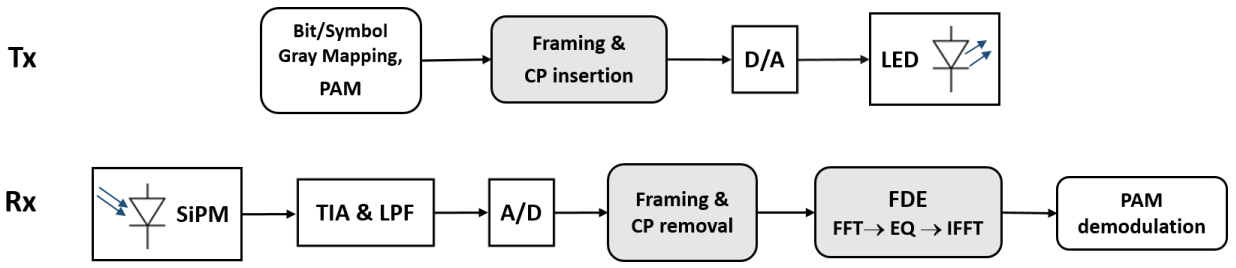

Fig. 3. Block diagram of the UWOC link using FDE at the Rx.

As explained in the previous section, to reduce the ISI effect due to limited component BWs at relatively high data rates, we perform FDE at the Rx. Consider Fig. 3 where at the Tx, we arrange the symbols in frames of size $N$ while inserting a cyclic prefix $(\mathrm{CP})$ at the end of each frame. The $\mathrm{CP}$ length should be equal to that of the aggregate channel $(\mathrm{AgC})$ impulse response (IR), that includes the IRs of the channel and $\mathrm{Tx} / \mathrm{Rx}$ components. At the Rx, after photo-detection and $\mathrm{A} / \mathrm{D}$, we remove the $\mathrm{CP}$ from each frame of received signals and then proceed to FDE. This equalization is realized by taking a $N$-point fast-Fourier-transform (FFT) of the received signal block, a single-tap zero-forcing equalization (EQ), followed by an inverse FFT (IFFT) to return back to the time domain (the reader is referred to see [22] for more details). Afterwards, PAM demodulation is done on the equalized signals. As discussed in the following section, we will refer to this modulation/demodulation approach as equi-distant (ED) PAM signaling, which is suboptimal for use in our quantum-noiselimited system.

\section{Signal Transmission and Detection}

Using uncoded NRZ $M$-ary PAM ( $M$-PAM), denote the highest and lowest transmit intensity levels by $P_{\max }$ and $P_{\min }$, respectively, and define the extinction ratio $\mathrm{EXT}=P_{\max } / P_{\min }$. Note that $\mathrm{OOK}$ is a special case with $M=2$.

\section{A. ED PAM Signaling}

At the Tx, $M$ ED power levels are used. This way, the PAPR is the same, whatever $M$. Signal demodulation can be based on the general approach of likelihood ratio test or by using a detection threshold $\mu_{\text {th }}$ minimizing the error probability. As mentioned previously, given the limitation of the dead-time and also SiPM output saturation for relatively high received intensity levels (corresponding to short link distances), the distribution of the detected photo-electrons can be very different from a Poisson (which applies to the received photons on the SiPM surface) [28]. Based on [28], it can be shown that in our application, this is not really constraining because the concerning link ranges are too short (e.g., less than one meter for a peak optical power of $1 \mathrm{~W}$ ). Consequently, we use the approximation of Poisson PDF for the detected electrons.

For instance, for the case of OOK modulation, corresponding to On and Off symbols, we have Poisson distributions with mean SiPM output counts of $\mu_{\mathrm{PQ}, 1}$ and $\mu_{\mathrm{PQ}, 0}$, respectively. The optimal threshold $\mu_{\mathrm{th}}$ in the sense of maximum likelihood results in the same error probability on bits zero and one. It can be easily shown that $\mu_{\text {th }}$ is given by: ${ }^{1}$

$$
\mu_{\mathrm{th}}=\left(\mu_{\mathrm{PQ}, 1}-\mu_{\mathrm{PQ}, 0}\right) / \log \left(\mu_{\mathrm{PQ}, 1}-\mu_{\mathrm{PQ}, 0}\right) .
$$

For $M>2$, signal detection can be done using $\left(\log _{2} M-1\right)$ detection threshold levels using similar calculations, i.e., resulting in the same error probability for two adjacent symbols in the signal constellation. This way, the $i$-th detection threshold $\mu_{\mathrm{th}, i}$ is obtained as follows:

$$
\mu_{\mathrm{th}, i}=\left(\mu_{\mathrm{PQ},(i+1)}-\mu_{\mathrm{PQ}, i}\right) / \log \left(\mu_{\mathrm{PQ},(i+1)}-\mu_{\mathrm{PQ}, i}\right),
$$

where, $\mu_{\mathrm{PQ}, i}$ denotes the corresponding $i$-th mean signal level. However, using these thresholds for signal demodulation is not optimal in our case, as we explain in the following.

\section{B. Optimal PAM Signaling}

Recall that in our case the dominant noise source at the $\mathrm{Rx}$ is the quantum (shot) noise whose variance depends on the received signal intensity [31]. As a result, the optimality of the detection thresholds presented above is only valid for the case of 2-PAM. This is due to the fact that the receiver noise has a higher variance for higher PAM levels where errors are more likely to occur. This effect can be seen in Fig. 4 that shows typical histograms of the received photon counts for the case of 4-PAM signaling.

For a more efficient signaling for $M>2$, we use the so-called square-root transformation (SQRT), proposed in [32], which allows for the normalization of the Poisson noise variance. In this manner, the shot-noise distorted $\mathrm{Rx}$ is transformed to a Rx which is affected by a white Gaussian signal-independent noise. Using this simple transformation, we can avoid non-uniform signaling that needs rather complex optimization for calculating the signal constellation [33], [34]. As shown in the block diagram of Fig. 5, we apply SQRT to the received symbols, and likewise, we do the inverse SQRT at the Tx [32]. Then, classical signal modulation and demodulation can be done at the Tx and at the Rx as for the case of a classical additive white Gaussian noise (AWGN) channel. In other words, we use ED PAM levels at the Tx before inverse SQRT, and use ED threshold levels at the Rx after SQRT. Let us denote the symbols at the input and output of the "Inverse Square-Root Transform" block in Fig. 5 by $x$ and $x_{\mathrm{sq}}=x^{2}$,

\footnotetext{
${ }^{1}$ As explained above, for very short ranges, the SiPM output cannot accurately be modeled as Poisson-distributed. For such ranges, the simple threshold-based demodulation is not optimal. However, under such conditions, the communication link is of poor quality and little data can be conveyed (see numerical results in Section V). We accordingly exclude such cases in our Rx design.
} 
Tx
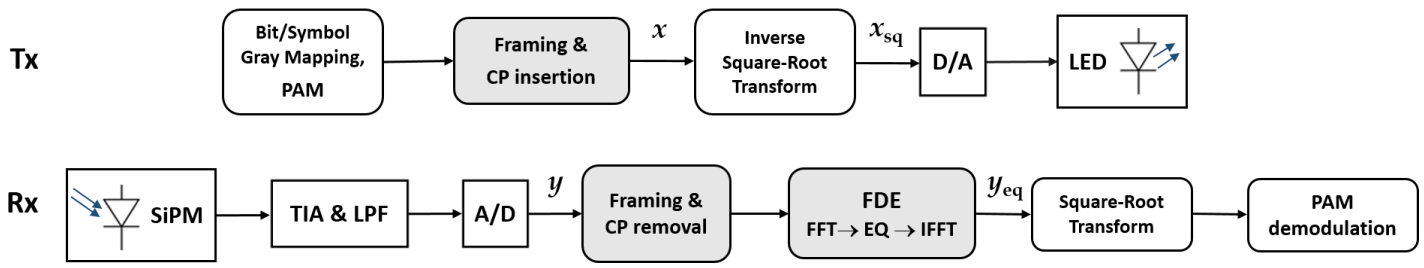

Fig. 5. Block diagram of the UWOC link using inverse SQRT and SQRT processing at the Tx and the Rx, respectively.

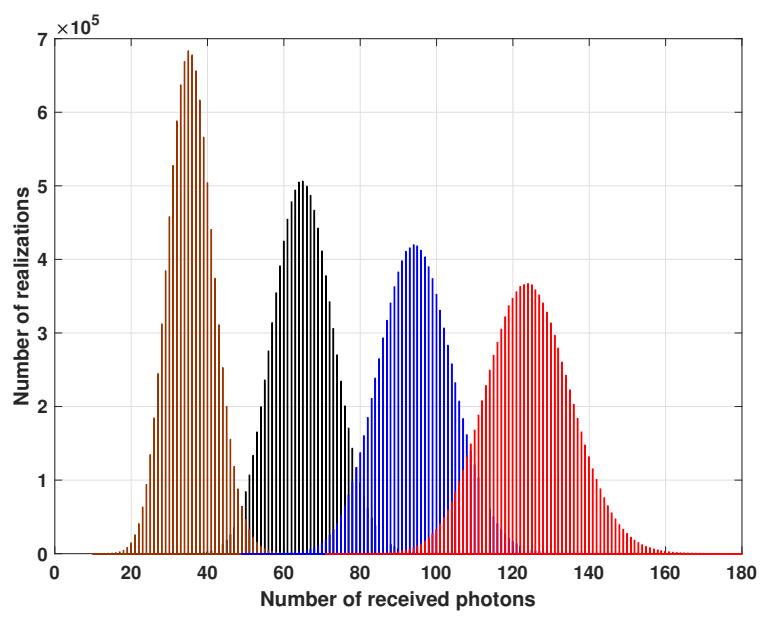

Fig. 4. Example of histogram of detected photo-electrons at the output of a PQ SiPM for the case of 4-PAM signaling, $1 \mathrm{Mbps}$ data-rate, MicroSB$30020 \mathrm{SiPM}, Z=67.5 \mathrm{~m}, P_{\mathrm{av}}=0.6 \mathrm{~W}$, based on the transmission of $10^{7}$ equi-probable 4-PAM symbols.

respectively. We also denote the received signal samples (at the A/D output) by $y$. Thus,

$$
y=y_{0}+\mathrm{n} \quad ; \quad y_{0}=\alpha x_{\mathrm{sq}} * h_{\mathrm{AgC}},
$$

where $*$ is the convolution operator, $h_{\mathrm{AgC}}$ stands for the normalized IR of the $\mathrm{AgC}$, and $\alpha$ is a constant that includes the channel loss factor and signal weighting at the Tx/Rx. Also, $n$ denotes the quantum noise. The variance of each sample of $n$ is proportional to the corresponding sample of $y_{0}$. Then, (linear) channel equalization is done on $y$ before applying SQRT (the linear convolution model of (6) is valid before SQRT). Now, denoting the output of the FDE block by $y_{\text {eq }}$, we equivalently consider the effect of the ensemble of the CP removal and FDE as that of an equivalent time-domain equalizer of IR $h_{\mathrm{AgC}}^{\mathrm{inv}}$, where $h_{\mathrm{AgC}}(n) * h_{\mathrm{AgC}}^{\mathrm{inv}}(n) \approx \delta(n)$. Consequently,

$$
y_{\mathrm{eq}}=y * h_{\mathrm{AgC}}^{\mathrm{inv}}=\alpha x_{\mathrm{sq}}+\mathrm{n}_{\mathrm{eq}}
$$

where, $\mathrm{n}_{\mathrm{eq}}=\mathrm{n} * h_{\mathrm{AgC}}^{\mathrm{inv}}$. Note that the statistics of $\mathrm{n}$ in the frequency domain are not straightforward to model. For the sake of simplicity, for the analysis of FDE, we do the same as if we were concerned with a signal-independent AWGN channel, although $\mathrm{n}$ is not independent from $y_{0}$. Although this is suboptimal in the sense of channel equalization, we will show that it still allows a significant improvement in the $\mathrm{Rx}$ performance (designing optimal FDE is beyond the scope of this paper).

Lastly, the inverse SQRT at the Tx obviously increases the signal PAPR. However, as we will see in the next section, this remains quite moderate for the case of $M$-PAM signaling.

\section{Link PERFormance Study}

Based on the mathematical model and formulation presented in the previous sections, we present here a set of simulation results to study, in particular, the effect of ISI on the link performance when increasing the data rate, performance improvement offered by FDE, the NLD effect of the SiPM, and the real interest of using non-binary PAM for SiPM-based Rxs.

\section{A. Assumptions and parameter specification}

At the Tx, we consider a blue LED at $\lambda=470 \mathrm{~nm}$, e.g., the NICHIA NSPB510AS model [35]. For the reference case of ED PAM signaling, we fix EXT to 0.2 that permits fast switching of the LED. Unless otherwise mentioned, we set the peak optical power $P_{\max }$ to $1 \mathrm{~W}$, which corresponds to an average transmit power of $0.6 \mathrm{~W}$ and a PAPR of 1.667 (irrespective of $M$ ). We assume that we work in the linear LED operation range and do not consider any clipping effect at the Tx. This assumption is quite justified as we will consider PAM levels up to $M=16$ (at most), resulting in a moderate PAPR, i.e., less than 2.5.

At the Rx, we consider two different SiPM components from ON Semiconductor ${ }^{\circledR}$ 's SensL B-series fast blue-sensitive products [36]: MicroSB-30020 (with a relatively large $N_{\text {SPAD }}$ ) and MicroSB-30035 (with a moderate $N_{\text {SPAD }}$ ) with the detailed parameters provided in Table I, corresponding to an overvoltage $V_{\mathrm{OV}}=2.5 \mathrm{~V}$ and when the "standard output" (anodeto-cathode readout) of the SiPM is used. No concentrating lens is used at the $\mathrm{Rx}$ to ensure a large $\mathrm{Rx}$ field-of-view (FOV), close to $180^{\circ}$, which allows better dealing with the beam misalignments [37]. Additionally, assume perfect channel knowledge and perfect time synchronization at the Rx, and no channel coding is used (see [38] on the interest of channel coding for UWOC links).

The BW limitation of the LED and the SiPM are modeled by first-order LPFs [8] of 3-dB cut-off frequencies $f_{\mathrm{c}, \mathrm{L}}$ and $f_{\mathrm{c}, \mathrm{S}}$, respectively. For the considered LED, $f_{\mathrm{c}, \mathrm{L}} \approx 10 \mathrm{MHz}$ [8]. Also, according to [36], $f_{\mathrm{c}, \mathrm{S}} \approx 2 \mathrm{MHz}$ for MicroSB-30035 (when using the standard output). The identical value is used for MicroSB-30020 since it is not reported on the data sheet.

\section{B. Effect of ISI and Practical Interest of FDE}

Let us firstly focus on the case of 2-PAM. As the symbol rate is increased (i.e., $T$ decreased), the link performance will be increasingly affected by ISI resulting from the limited 
TABLE I

SIPM PARAMETERS

\begin{tabular}{|c|c|c|}
\hline Parameter & MicroSB-30020 & MicroSB-30035 \\
\hline 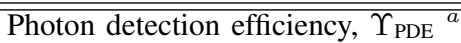 & $\overline{24 \%}$ & $\overline{31 \%}$ \\
\hline Gain $G^{a}$ & $10^{6}$ & $3 \times 10^{6}$ \\
\hline Surface area & $9 \mathrm{~mm}^{2}$ & $9 \mathrm{~mm}^{2}$ \\
\hline Microcell size & $20 \mu \mathrm{m}$ & $35 \mu \mathrm{m}$ \\
\hline Fill factor $\alpha_{\mathrm{FF}}$ & $49 \%$ & $65 \%$ \\
\hline Dark count rate, $f_{\mathrm{DCR}}{ }^{a}$ & $6.6 \mathrm{MHz}$ & $6.7 \mathrm{MHz}$ \\
\hline Dead-time, $\tau_{\mathrm{d}}{ }^{a}$ & $100 \mathrm{~ns}$ & $180 \mathrm{~ns}$ \\
\hline Number of SPADs, $N_{\mathrm{SPAD}}$ & 10998 & 4774 \\
\hline Probability of cross-talk, $P_{\mathrm{CT}}{ }^{a}$ & $3 \%$ & $7 \%$ \\
\hline Probability of after-pulsing, $P_{\mathrm{AP}}{ }^{a}$ & $0.2 \%$ & $0.2 \%$ \\
\hline
\end{tabular}

${ }^{a}$ Corresponding to typical values provided in the data-sheet [36] for an overvoltage of $2.5 \mathrm{~V}$.

BW of the Tx/Rx components. To quantify the extent of the degradation, we have presented in Fig. 6 BER plots for different data rates. For the bit rate of $1 \mathrm{Mbps}$, the limited BWs of the LED and the SiPMs have negligible effect on the link performance.

Let consider relatively low data rates, i.e., the cases of 1,2 , and $5 \mathrm{Mbps}$. We notice a better performance for the MicroSB30035 detector, which is due to its larger gain, or in other words, its higher PDE, compared to the 30020 model (see Table I). For instance, for $1 \mathrm{Mbps}$ bit-rate and a target BER of $10^{-6}$, the link span is limited to $\approx 65.5 \mathrm{~m}$ with the former, compared to $\approx 61.5 \mathrm{~m}$ for the latter. However, having a smaller number of microcells $N_{\text {SPAD }}$, the 30035 detector is more subject to NLD at relatively short ranges: the limit of detector saturation being around $Z \approx 2.5 \mathrm{~m}$, compared to $1 \mathrm{~m}$ for the 30020 model. $^{2}$

In order to better clarify the DR limitation, let us for simplicity neglect the effects of crosstalk, after-pulsing, and dark current. Then, according to (2), the average number of received photons is $\mu \approx \Upsilon_{\mathrm{PDE}} P_{\mathrm{Rx}} T / E_{\mathrm{ph}}$. If again for simplicity, we assume that the actual photon count $\mu_{a} \approx \mu$, from (3), the reduction in the average photon count at the output of the PQ SiPM, denoted here by $\psi$ is,

$$
\psi=\exp \left(-\frac{\Upsilon_{\mathrm{PDE}} P_{\mathrm{Rx}} \tau_{\mathrm{d}}}{E_{\mathrm{ph}} N_{\mathrm{SPAD}}}\right) .
$$

It is important to notice that $\psi$ does not depend on $T$, and hence, it is independent of the transmission rate. At large $Z$ values, the term in the exponential becomes very small and, therefore, $\psi \rightarrow 1$. At relatively short $Z$, however, where $P_{\mathrm{Rx}}$ becomes relatively high, $\psi$ causes an important loss in $\mu_{\mathrm{PQ}}$. For instance, for $Z=2$ and $10 \mathrm{~m}$ corresponding to channel losses of $\sim 66.7$ and $\sim 79 \mathrm{~dB}$ (see Fig. 2 ), we have $\psi=0.23$ and 0.92 for 30020 detector, and $\psi=3.8 \times 10^{-4}$ and 0.63 for 30035 detector, respectively.

Consider next the case of relatively high data rates $(\geq$ $10 \mathrm{Mbps}$ ) where the severe ISI effect results in $\mathrm{BER} \approx 0.5$ (without equalization). When FDE is performed, a substantial

${ }^{2}$ Recall from Section III that the statistical model that we considered is not valid for those short ranges. However, the BER is too high at these short ranges due to SiPM saturation, and hence, the exact statistical model has little importance. improvement can be achieved in the link performance. Nevertheless, an increased data rate results in a reduction in the maximum link range for a given target BER. For instance, for MicroSB-30020 detector and a target BER of 10-6 (Fig. 6(a)), the link span is limited to around $41.5,32.5,20.8$, and $4 \mathrm{~m}$ for bit-rates of $5,10,20$, and $50 \mathrm{Mbps}$. This can be explained by the fact that, for higher data rates, the link suffers from a more significant frequency selectivity. Although FDE helps reduce the ISI effect, obviously, we do not attain the performance of an ideal link. It is worth mentioning that the ZF equalization that we consider here results in noise amplification at relatively high frequencies where the LPF transfer function of the $\mathrm{AgC}$ considerably attenuates the signal power. Also, remember from Section IV-B that we have considered an approximate suboptimal formulation for the equalizer. At very high data rates, here for $50 \mathrm{Mbps}$, the joint effect of increased noise level (due to ZF FDE) and decreased effective electrical signal power (i.e., a small $\psi$ ) results in a more noticeable performance degradation at relatively short $Z$. This is in particular the case for the 30035 model which supports a lower DR.

\section{Link range limitation versus modulation order for M-PAM}

Let us now investigate the limitation on the transmission range $Z$ for the general case of non-binary PAM. At first, we consider the classical case of ED PAM signaling for which we have presented in Fig. 7 BER as a function of $Z$ for two SiPM components. Here, similar to the case of 2-PAM in Fig.6, we set EXT and $P_{\max }$ to 0.2 and $1 \mathrm{~W}$, respectively. Also, the symbol rate is fixed to $1 \mathrm{Msps}$ (symbols per second), i.e., $T=$ $1 \mu \mathrm{s}$ at which the link suffers from practically no ISI (i.e., BER plots coincide with those under infinite $f_{\mathrm{c}, \mathrm{L}}$ and $\left.f_{\mathrm{c}, \mathrm{S}}\right)$. As expected, by increasing $M$, more signal-to-noise ratio (SNR) is required for signal detection, and the link span is accordingly decreased. We notice that due to the quantum-noise-limited $\mathrm{Rx}$, we have a rather drastic decrease in the attainable $Z$ by increasing $M$ for a target BER (compared with [21]).

Now let us consider the case of PAM transmission with SQRT processing. Due to inverse SQRT done at the Tx, see Fig. 5, we cannot guarantee the same EXT as in the case of classical PAM. We hence relax the condition on EXT (or in other words on $P_{\min }$ ) while keeping $P_{\mathrm{av}}=0.6 \mathrm{~W}$. The PAPR will be a bit higher compared to the previous case, however. 


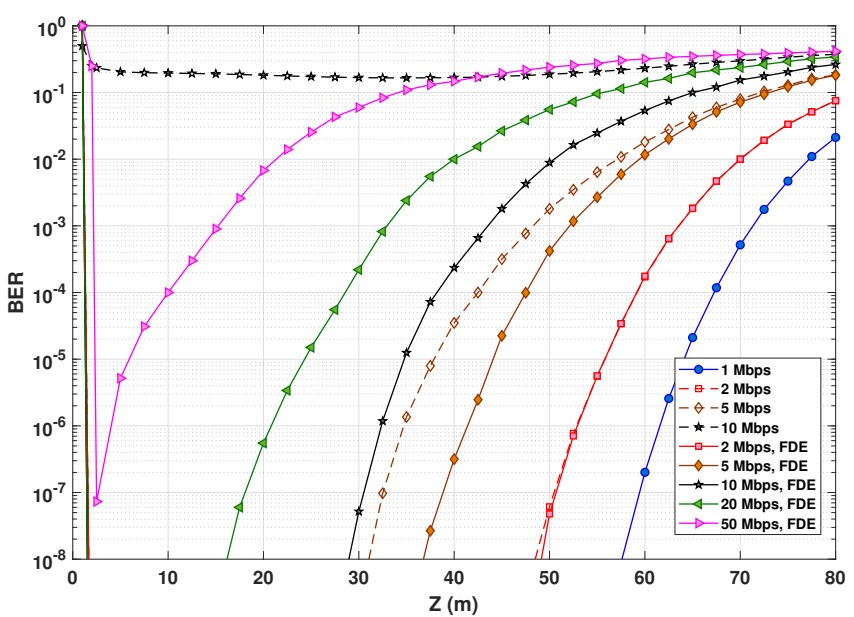

(a) MicroSB-30020

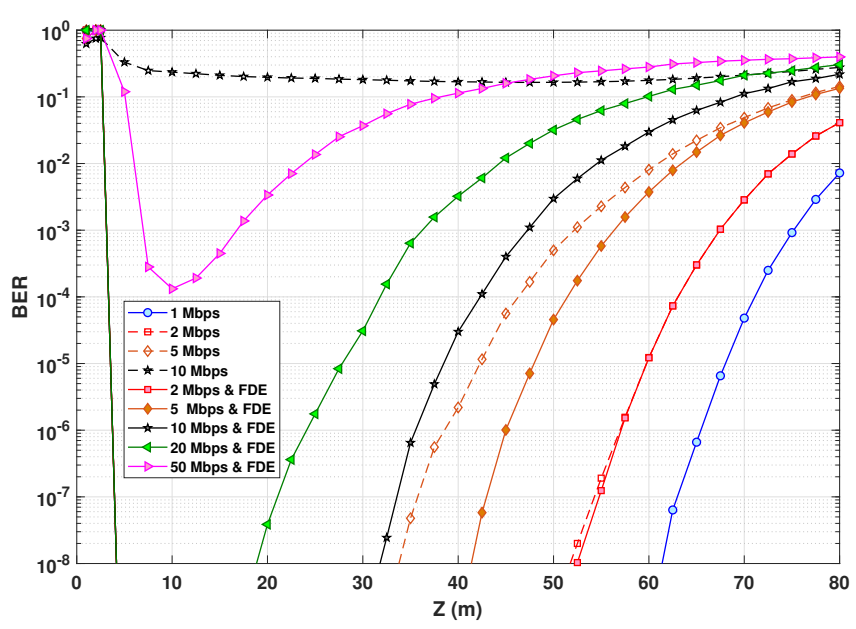

(b) MicroSB-30035

Fig. 6. BER versus $Z$ for different bit-rates with and without FDE at the Rx. Uncoded 2-PAM with EXT $=0.2$ (without $\mathrm{SQRT}$ ), clear ocean waters. Block size of $N=256$. CP is set to $2,5,6,8$, and 12 for data rates 2,5 , 10, 20, and $50 \mathrm{Mbps}$, respectively, adjusted to avoid inter-block interference. No equalization is necessary for $1 \mathrm{Mbps}$.

Although SQRT is not necessary for the case of 2-PAM, to make a fair comparison with higher $M$, we apply also this transformation to this modulation (EXT will then be different from in Fig. 6). The corresponding BER plots are presented in Fig. 7. Note that the plots for the two cases of ED and SQRT PAM should not be compared directly. Indeed, although we have the same $P_{\mathrm{av}}$ in both cases, PAM alphabet levels have a larger distance for the case of SQRT. This can be seen from Table II, where we have provided the transmit symbols for the two cases of classical and SQRT PAM signaling for $M=2$ and 4 together with the corresponding PAPR. The PAPR at the Tx for the case of SQRT processing is 1.9231, 2.2277, 2.333, and 2.3784 for $M=2,4,8$, and 16 , respectively.

From Fig. 7 we can see that irrespective of $P_{\min }$, SQRT processing allows a significant improvement in the link performance for $M>2$. Meanwhile, we notice that even the slight increase in the signal PAPR increases the minimum $Z$ before the saturation of the SiPM. This is more noticeable for

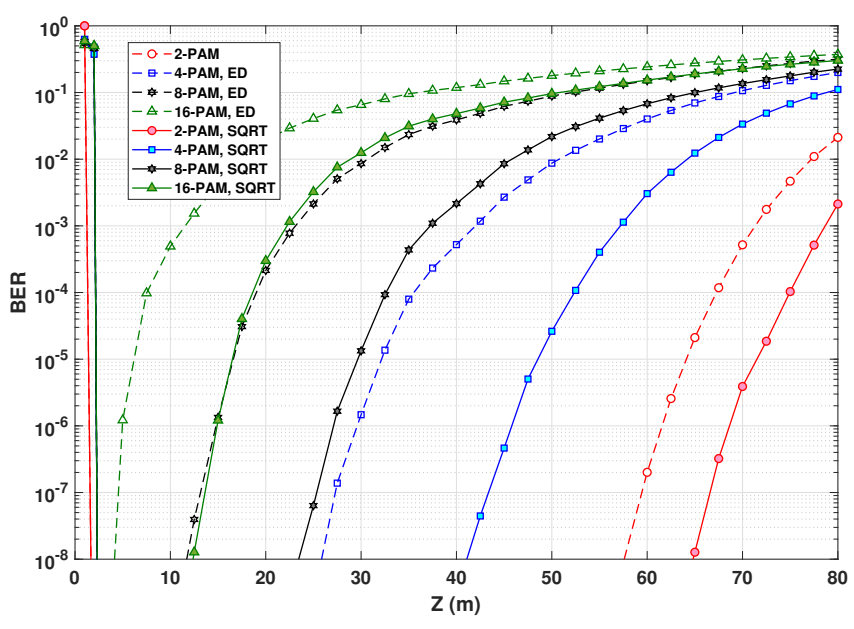

(a) MicroSB-30020

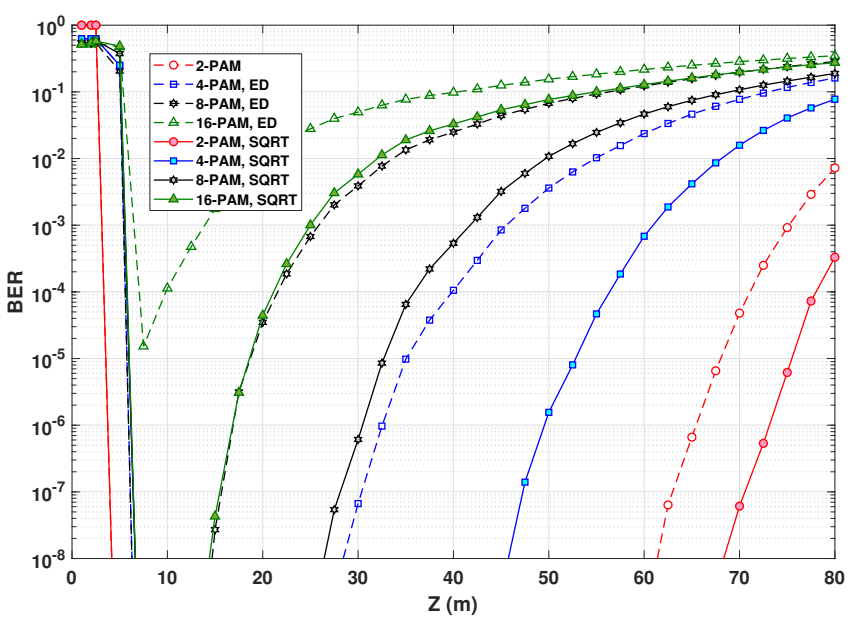

(b) MicroSB-30035

Fig. 7. BER versus link distance $Z$, uncoded $M$-PAM modulation with extinction factor EXT $=0.2$ for the cases of ED-PAM and PAM with SQRT processing. Symbol rate of $1 \mathrm{Msps}$ corresponding to $R=1,2,3,4 \mathrm{Mbps}$, for $M=2,4,8,16$, respectively. Clear ocean waters. Cases of "2-PAM" and "2-PAM, SQRT" refer to $P_{\min }=0.2$ and 0.0462 , respectively, see Table II.

the $30035 \mathrm{SiPM}$, which has a smaller $N_{\mathrm{SPAD}}$, hence tolerating a smaller DR, or in other words, a smaller PAPR.

In order to see the impact of the NLD limitation on the link performance for higher Tx power levels, we have presented in Fig. 8 BER plots for the case of PAM signaling with SQRT and $P_{\mathrm{av}}=6 \mathrm{~W}$ (i.e., 10 times increase with respect to the previous case ${ }^{3}$ ) for both SiPM models. The symbol rate is still set to 1 Msps where we can neglect the BW limitation of the LED and SiPM. Comparing these results with those in Fig. 7, we notice the significant increase in the maximum attainable range, but at the same time, in the minimum operation range of the Rx, especially for $M>2$. For instance, considering nonbinary PAM and a BER of $10^{-6}$, the minimum $Z$ is more than

\footnotetext{
${ }^{3}$ In practice, the increase in the transmit power can be achieved using multiple LEDs at the Tx, see for instance the use of 100 LEDs in a recent prototype in [9]. This way, the response time of the Tx is not affected, as compared to the case of using a high-power LED with a higher parasitic capacitance.
} 
TABLE II

TRANSMIT PAM SIGNAL LEVELS AND THE CORRESPONDING PAPR

\begin{tabular}{|c|c|c|c|c|c|c|c|}
\hline & \multicolumn{3}{|c|}{ ED } & $P A P R$ & \multicolumn{2}{|c|}{ SQRT } & $P A P R$ \\
\hline 2-PAM & & 0.2 & 1 & 1.667 & 0.0462 & 1.1538 & 1.9231 \\
\hline 4-PAM & 0.2 & 0.4667 & $\begin{array}{ll}0.7333 & 1.0\end{array}$ & 1.667 & 0.0535 & 0.7188 & 2.2277 \\
\hline
\end{tabular}

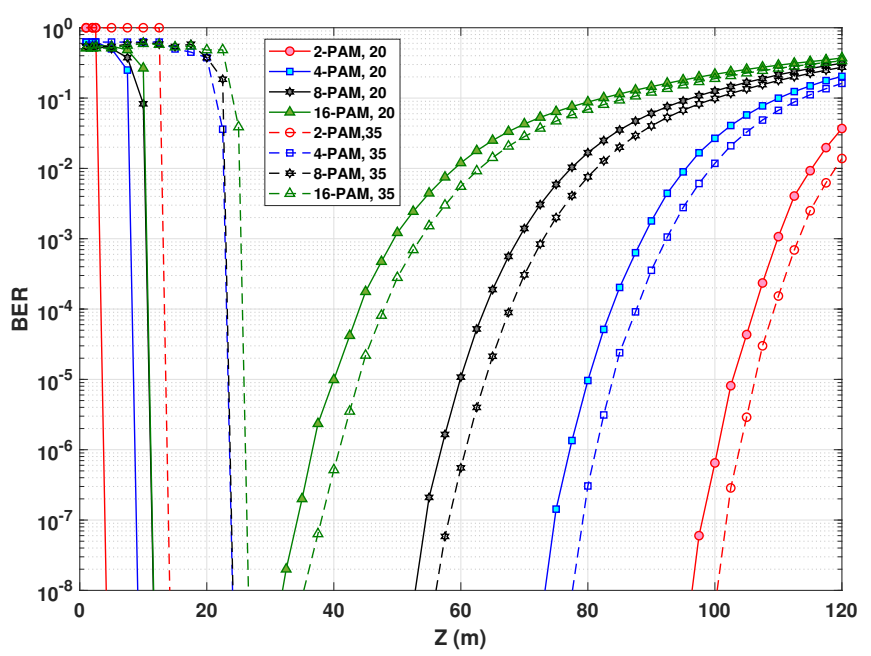

Fig. 8. BER versus $Z$, uncoded $M$-PAM modulation with $\mathrm{EXT}=0.2$ when SQRT is performed on the transmit PAM symbols. Symbol rate of 1 Msps corresponding to $R=1,2,3,4 \mathrm{Mbps}$, for $M=2,4,8,16$, respectively. Clear ocean waters. $P_{\mathrm{av}}=6 \mathrm{~W} .20$ and 35 on the figure legend refer to MicroSB-30020 and MicroSB-30035 components, respectively.

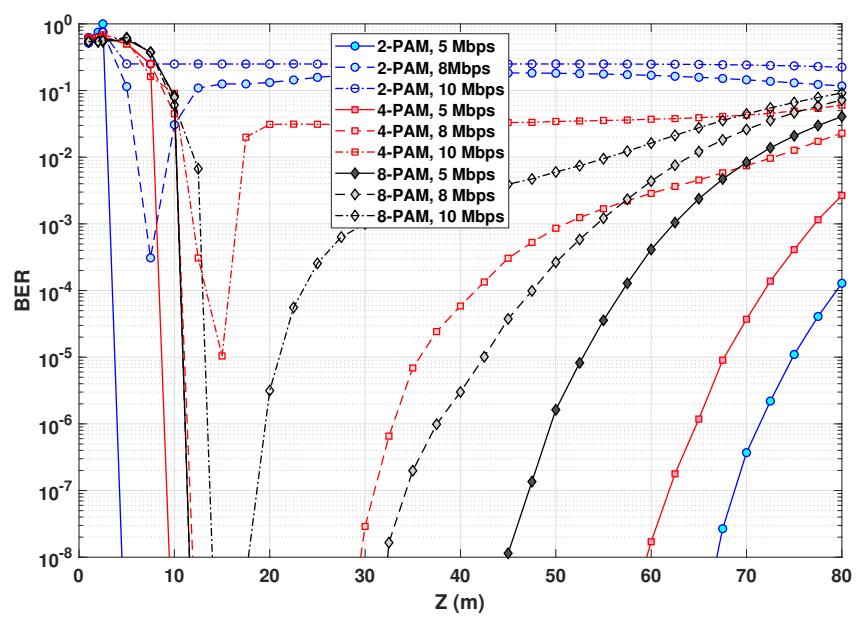

Fig. 9. BER versus link distance $Z$ for data rates of 5,8 , and 10 Mbps when no FDE is performed at the Rx. Uncoded $M$-PAM, $M=2,4,8$, with SQRT, MicroSB-30020 SiPM, clear ocean waters, $P_{\mathrm{av}}=6 \mathrm{~W}$.

8 and $24 \mathrm{~m}$ for 30020 and 30035 SiPMs, respectively.

\section{M-PAM and FDE}

To see the interest of using non-binary PAM for increasing the data rate, we study the BER performance for 2,4 , and 8-PAM modulations using SQRT and the MicroSB-30020 detector. At first, we perform no channel equalization at the $\mathrm{Rx}$, e.g., for the reasons of reducing implementation complexity.

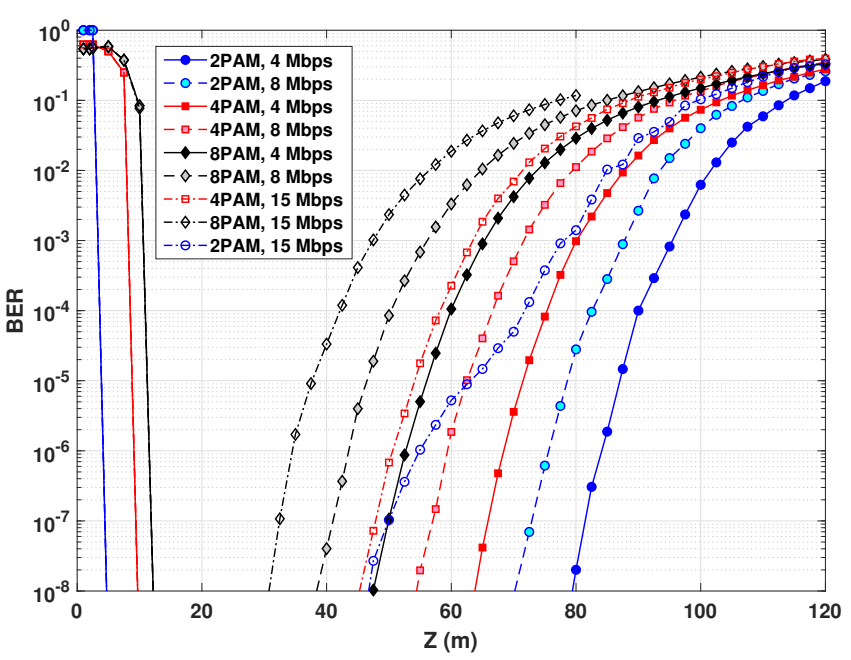

(a)

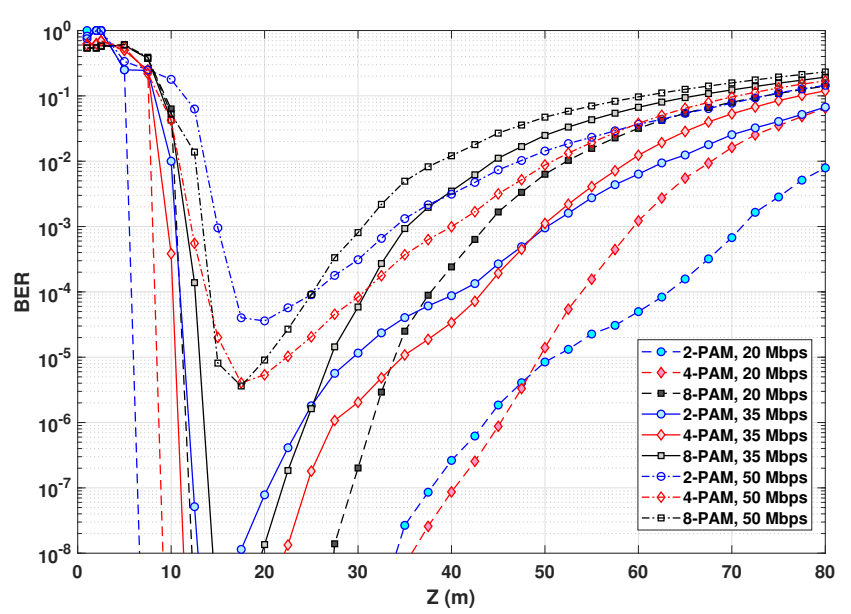

(b)

Fig. 10. BER versus link distance $Z$ for data rates of (a) $4,8,15 \mathrm{Mbps}$, and (b) 20,35, $50 \mathrm{Mbps}$ with FDE at the Rx. Uncoded 2, 4, and 8-PAM modulation with SQRT, MicroSB-30020 SiPM, clear ocean waters, $P_{\mathrm{av}}=$ $6 \mathrm{~W}$, block size $N=256$ symbols. For instance, CP is set to $4,6,7,8,10$, and 12 for 2-PAM and data rates $4,8,15,20,35$, and $50 \mathrm{Mbps}$, respectively.

We have presented the BER plots for the data rates of 5,8 , and $10 \mathrm{Mbps}$ in Fig. 9. As expected, by increasing the data rate, the ISI effect becomes increasingly important and penalizes the BER performance. Meanwhile, we notice the interest of using non-binary PAM, except for the relatively low data rate of $5 \mathrm{Mbps}$ where the ISI effect is not severe enough. This is due to the higher BW efficiency of non-binary PAM, compared with binary PAM. The advantage of 4- and 8-PAM over 2PAM for data rates of 8 and $10 \mathrm{Mbps}$ is indisputable.

Let us consider now the case where FDE is performed at 


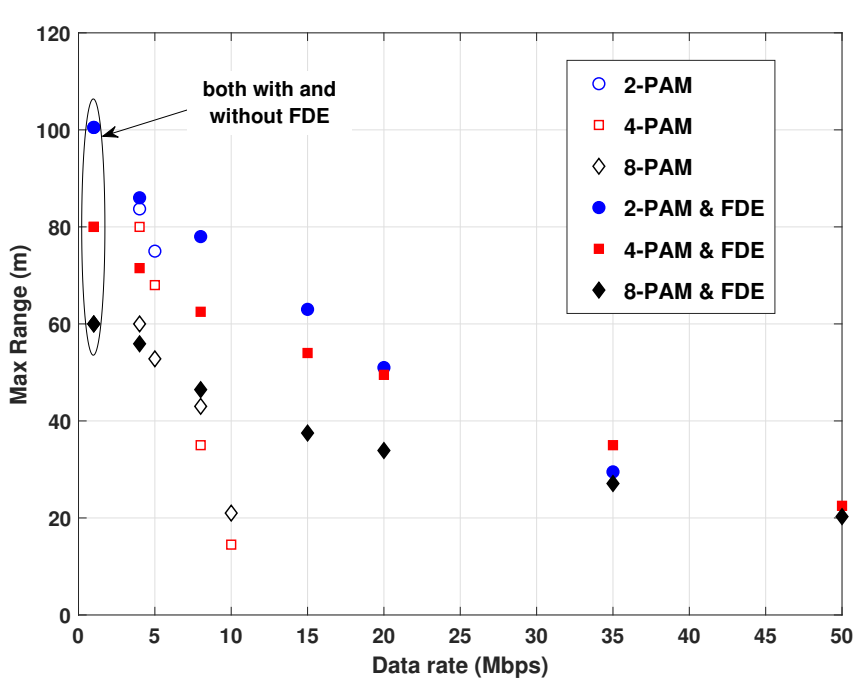

Fig. 11. Scatter plots of the maximum attainable link span for a given data rate and subject to BER $<10^{-5}$. Uncoded $M$-PAM with SQRT, MicroSB$30020 \mathrm{SiPM}$, clear ocean waters, $P_{\mathrm{av}}=6 \mathrm{~W}$.

the Rx. We have presented the BER plots versus $Z$ in Fig. 10 for the data rates 4, 8, and $15 \mathrm{Mbps}$ in Fig. 10(a) and 20,35, and $50 \mathrm{Mbps}$ in Fig. 10(b). The latter considers relatively high data rates with a more significant impact of ISI. We firstly notice from Fig. 10(a) that for "moderate" data rates, 2-PAM remains the most efficient scheme. However, it is seen that for the data rate of $15 \mathrm{Mbps}$, the performance of 2-PAM becomes severely affected, due to significant AgC frequency selectivity. If we consider the data rate of $20 \mathrm{Mbps}$, which corresponds to symbol rates of 20,10 , and $6.7 \mathrm{Msps}$ for the cases of 2,4 , and 8-PAM, respectively, we notice from Fig. 10(b) that there is no clear advantage of using non-binary PAM except for ranges below $\approx 48 \mathrm{~m}$ where 4 -PAM performs better. For $35 \mathrm{Mbps}$ data rate, the $Z$ interval where 4-PAM outperforms 2-PAM is larger, and for $50 \mathrm{Mbps}$, it almost always outperforms 2-PAM. Meanwhile, the advantage of 8-PAM is not really convincing, which can be explained by the fact that for a fixed $P_{\mathrm{av}}, 8-\mathrm{PAM}$ is more subject to demodulation errors than 4- and 2-PAM.

We have summarized the results of Figs. 9 and 10 in the scatter plot of Fig. 11, which shows the maximum attainable link span versus a desired data rate for a target BER of (at most) $10^{-5}$. Hollow and filled markers represent the case of PAM signaling without and with FDE at the Rx, respectively. The absence of marker for a given data rate signifies that the modulation scheme cannot be used, i.e., the BER will exceed $10^{-5}$. This is, for instance, the case for 2-PAM (without FDE) at data rates larger than $5 \mathrm{Mbps}$, where we can clearly see the interest of using 4- and 8-PAM. When performing FDE, non-binary PAM schemes become advantageous at very high data-rates, where the $\mathrm{AgC}$ becomes highly frequency selective, here for data rates higher than $\sim 20$ Mbps.

\section{CONCLUSIONS AND Discussions}

We considered the use of non-binary PAM modulation for UWOC links where an SiPM is used at the Rx for signal detection. We presented a detailed formulation for the transmission link that took into account the parameters of the Tx, the Rx, and the aquatic channel, while assuming that this latter does not introduce any pulse broadening. We focused on the limitation on the data rate due to the limitations on the LED and the SiPM BW, as well as on the SiPM DR. We showed how a detector with a larger number of SPADs helps work in relatively short ranges, especially for relatively high transmit powers (which is usually the case in UWOC systems) as well as for using larger modulation orders $M$. The substantial performance improvement achieved by channel equalization in the Rx was shown for symbol rates much higher than the LED/SiPM BWs, where the induced ISI can render the link nonoperational. The considered channel equalization can be further optimized, in particular, to account for the signal dependent noise, which is the subject of on-going research.

Considering non-binary PAM modulation, we also elucidated the interest of using SQRT processing to deal with the signal-dependent quantum noise at the $\mathrm{Rx}$. We also showed that using non-binary PAM is advantageous as compared with 2-PAM only for relatively high data rates, where the communication link can suffer from severe ISI. Note that we considered in this paper the case of uncoded modulation. The performance gain will be more considerable when error correction coding is performed [38].

In the following, we discuss some other important points regarding the presented study.

- Achievable data-rate:

We considered in this study data rates up to $50 \mathrm{Mbps}$; the main limitation arising from the limited BW of the LED and the SiPM. Higher data rates can be readily attained by using LEDs with a larger BW or ultimately laser diodes at the Tx. Note that all over-Gbps UWOC links reported in the recent literature, e.g., [10], [11], consider the use of lasers at the Tx. The Rx BW can also be increased by using SiPMs with a smaller active area, yet, resulting in a more significant geometric loss, or equivalently, a reduced link span for a target BER.

- Achievable transmission range:

The achievable maximum transmission range in the presented results may seem rather moderate. Longer spans can be attained with higher Tx powers, e.g., by using multiple LEDs (rather than using a high-power LED that could have a slow response). This way we can obtain a high transmit power while keeping a fast switched Tx. For instance, for an average transmit power of $100 \mathrm{~W}$, a link span of about $152 \mathrm{~m}$ can be achieved for the 30020 detector for a bit rate of $1 \mathrm{Mbps}$ and a target BER of $10^{-4}$. Obviously, a larger received power can also be obtained by using a lens at the Rx, but this will limit the Rx FOV, necessitating more precise beam pointing and tracking. Similarly, concerning the emitter beam width, which was considered about $20^{\circ}$ in this paper, a tradeoff should be made in practice between relaxing beam pointing constraints and the attainable transmission range.

- Background noise effect:

Although we neglected the effect of background noise, it can severely affect the Rx performance due to the high 
sensitivity of SiPMs. In a recent study [39], the impact of solar background noise on the link performance was investigated for a worst-case scenario, i.e., sun at the zenith and the Rx plan directed towards the sea surface, this way, capturing the maximum solar irradiation [30]. It was shown that for the case of clear ocean waters and considering OOK modulation and the MicroSB-30020 SiPM model, for operation depths (with respect to the $\mathrm{Rx}$ ) larger than $\sim 250 \mathrm{~m}$ the link performance is not practically affected by the background noise [39]. This in fact sets the pessimistic minimum depth, beyond which the link is not subject to solar noise.

- Other simplifying conditions:

Another important assumption that we made concerned perfect time synchronization and perfect channel knowledge for signal demodulation at the Rx. Both synchronization and channel estimation can be done using some training sequences, which is rather classical in the context of wireless communications. For typical UWOC links using intensity modulation and direct detection and operating under quasi-static channel statistics, these tasks are even much simpler to realize. For instance, using a very negligible pilot overhead, an almost perfect channel estimate can be obtained (see [40], [41] or the similar case of free-space optics)

We also considered in the presented results some simplified conditions for the aquatic channel, e.g., clear ocean waters with relatively low scattering and absorption effects, and absence of water turbulence. The presented study remains nevertheless relevant in general. For instance, in the case of higher turbidity waters, FDE can remedy the effect of pulse broadening. Also, water turbulence and background noise effects can be reduced using diversity techniques and channel coding, respectively.

\section{REFERENCES}

[1] M. A. Khalighi, C. J. Gabriel, L. M. Pessoa, and B. Silva, Visible Light Communications: Theory and Applications. CRC-Press, 2017, ch. Underwater Visible Light Communications, Channel Modeling and System Design, pp. 337-372.

[2] S. Arnon, "Underwater optical wireless communication network," Optical Engineering, vol. 49, no. 1, pp. 1-6, Jan. 2010.

[3] H. Kaushal and G. Kaddoum, "Underwater optical wireless communication," IEEE Access, vol. 4, pp. 1518-1547, Apr. 2016.

[4] F. Hanson and S. Radic, "High bandwidth underwater optical communication," Applied Optics, vol. 47, no. 2, pp. 277-283, Jan. 2008.

[5] C. Gabriel, M. A. Khalighi, S. Bourennane, P. Léon, and V. Rigaud, "Monte-carlo-based channel characterization for underwater optical communication systems," IEEE/OSA J. Opt. Commun. Networking vol. 5, no. 1, pp. 1-12, Jan. 2013.

[6] B. Cochenour, L. Mullen, and J. Muth, "Temporal response of the underwater optical channel for high-bandwidth wireless laser communications," IEEE J. Oceanic Eng., vol. 38, no. 4, pp. 730-742, Oct. 2013

[7] W. Cox and J. Muth, "Simulating channel losses in an underwater optical communication system," Journal of the Optical Society of America A, vol. 31, no. 5, pp. 920-934, May 2014.

[8] M. A. Khalighi, T. Hamza, S. Bourennane, P. Léon, and J. Opderbecke, "Underwater wireless optical communications using silicon photo-multipliers," IEEE Photonics Journal, vol. 9, no. 4, 2017, dOI 10.1109/JPHOT.2017.2726565.

[9] P. Léon, F. Roland, L. Brignone, J. Opderbecke, J. Greer, M. A. Khalighi, T. Hamza, S. Bourennane, and M. Bigand, "A new underwater optical modem based on highly sensitive silicon photo-multipliers," IEEE OCEANS Conference, June 2017, Aberdeen, UK.
[10] H. M. Oubei, J. R. Duran, B. Janjua, H.-Y. Wang, C.-T. Tsai, Y.-C. Chi, T. K. Ng, H.-C. Kuo, J.-H. He, M.-S. Alouini, G.-R. Lin, and B. S. Ooi, "4.8 Gbit/s 16-QAM-OFDM transmission based on compact 450-nm laser for underwater wireless optical communication," Optics Express, vol. 23, no. 18, pp. 23 302-23 309, Sept. 2015

[11] K. Nakamura, I. Mizukoshi, and M. Hanawa, "Optical wireless transmission of $405 \mathrm{~nm}, 1.45 \mathrm{Gbit} / \mathrm{s}$ optical IM/DD-OFDM signals through a 4.8 m underwater channeln," Optics Express, vol. 23, no. 2, pp. 1558-1566, Jan. 2015.

[12] C. Gabriel, M. A. Khalighi, S. Bourennane, P. Léon, and V. Rigaud, "Investigation of suitable modulation techniques for underwater wireless optical communication," Int. Workshop Optical Wireless Commun. (IWOW), pp. 1-3, Oct. 2012, Pisa, Italy.

[13] Z. Ghassemlooy, A. Hayes, N. Seed, and E. Kaluarachchi, "Digital pulse interval modulation for optical communications," IEEE Commun. Mag., vol. 48, pp. 95-99, Dec. 1998

[14] Introduction to Silicon Photo-Multipliers (SiPMs). First Sensor, version 03-12-15, available online on http://www.first-sensor.com.

[15] N. Otte, "The Silicon Photomultiplier - a new device for high energy physics, astroparticle physics, industrial and medical applications," International Symposium on Detectors for Particle, Astroparticle and Synchrotron Radiation Experiments (SNIC), pp. 1-9, Apr. 2006, Stanford, California.

[16] S. Donati and T. Tambosso, "Single-photon detectors: From traditional PMT to solid-state SPAD-based technology," IEEE Journal of Selected Topics in Quantum Electronics, vol. 20, no. 6, pp. 204-211, Nov. 2014.

[17] A. Gallivanoni and M. G. I. Rech, "Progress in quenching circuits for single photon avalanche diodes," IEEE Transactions on Nuclear Science, vol. 57, no. 6, pp. 3815-3826, Dec. 2010

[18] Introduction to SiPM, Technical Note. ON Semiconductor ${ }^{\circledR} \quad$ - SensL, 2011 (Rev. 6.0, Feb. 2017) available at https://www.sensl.com/downloads/ds/TN\%20 \%20Intro\%20to\%20SPM\%20Tech.pdf.

[19] ID Quantique, "ID150 VIS 8 Channel SPAD : Miniature 8-channel photon counter for OEM applications ," http://www.idquantique.com, 2010.

[20] Y. Li, M. Safari, R. Henderson, and H. Haas, "Nonlinear distortion in SPAD-based optical OFDM systems," Global Communications Conference, Optical Wireless Communications Workshop, pp. 1-6, Dec. 2015, San Diego, CA.

[21] M. Wolf, S. A. Cheema, M. A. Khalighi, and S. Long, "Transmission schemes for visible light communications in multipath environments,' ICTON Conference, pp. 1-7, July 2015, Budapest, Hungary.

[22] M. A. Khalighi, S. Long, S. Bourennane, and Z. Ghassemlooy, "PAM and CAP-based transmission schemes for visible-light communications," IEEE Access Journal, Special issue on Optical Wireless Technologies for 5 G Communications and Beyond, vol. 5, pp. 27 002-27 013, Oct. 2017.

[23] S. Hranilovic, Wireless Optical Communication Systems. Springer, 2005.

[24] B. M. Cochenour, L. J. Mullen, and A. E. Laux, "Characterization of the beam-spread function for underwater wireless optical communications links," IEEE Journal of Oceanic Engineering, vol. 33, no. 4, pp. 513521, Oct 2008

[25] Y. Ata and Y. Baykal, "Structure functions for optical wave propagation in underwater medium," Waves in Random and Complex Media, vol. 24 no. 2, pp. 164-173, Feb. 2014

[26] Y. Baykal, "Scintillations of LED sources in oceanic turbulence," Applied Optics, vol. 55, no. 31, pp. 8860-8863, Nov. 2016.

[27] C. Gabriel, M. A. Khalighi, S. Bourennane, P. Léon, and V. Rigaud, "Channel modeling for underwater optical communication," IEEE Workshop on Optical Wireless Communications, Global Communication Conference, pp. 833-837, Dec. 2011, Houston, TX.

[28] E. Sarbazi, M. Safari, and H. Haas, "Statistical modeling of singlephoton avalanche diode receivers for optical wireless communications," IEEE Transactions on Communications, vol. Early Access, 2018.

[29] Y. Li, M. Safari, R. Henderson, and H. Haas, "Nonlinear distortion in SPAD-based optical OFDM systems," Global Communications Conference (GLOBECOM), Workshop on Optical Wireless Communications, Dec. 2015, San Diego, CA.

[30] T. Hamza, M. A. Khalighi, S. Bourennane, P. Léon, and J. Opderbecke, "Investigation of solar noise impact on the performance of underwater wireless optical communication links," Optics Express, vol. 24, no. 22, pp. $25832-25845$, Oct. 2016

[31] F. Xu, M. A. Khalighi, and S. Bourennane, "Impact of different noise sources on the performance of PIN- and APD-based FSO receivers," COST IC0802 Workshop, IEEE ConTEL Conference, pp. 211-218, June 2011, Graz, Austria. 
[32] M. Safari, "Efficient optical wireless communication in the presence of signal-dependent noise," International Conference on Communications (ICC), June 2015, London, UK.

[33] T. H. Chan, S. Hranilovic, and F. R. Kschischang, "Capacity-achieving probability measure for conditionally Gaussian channels with bounded inputs," IEEE Transactions on Information Theory, vol. 51, no. 6, pp. 2073-2088, June 2005.

[34] J. Cao, S. Hranilovic, and J. Chen, "Capacity and non-uniform signalling for discrete-time Poisson channels," IEEE/OSA Journal of Optical Communications and Networking, vol. 5, no. 4, pp. 329-337, Apr. 2013.

[35] NICHIA Corporation, "Specifications for blue LED: NSPB510AS," http://www.nichia.co.jp.

[36] B-Series Fast, Blue-Sensitive Silicon Photomultiplier Sensors Datasheet and User Manual. ON Semiconductor ${ }^{\circledR}$ - SensL, 2013, available at http://www.sensl.com/downloads/ds/UM-MicroB.pdf.

[37] C. Gabriel, M. A. Khalighi, S. Bourennane, P. Léon, and V. Rigaud, "Misalignment considerations on point-to-point underwater wireless optical links," IEEE OCEANS Conference, June 2013, Bergen, Norway.

[38] F. Mattoussi, M. Khalighi, and S. Bourennane, "Improving the performance of underwater wireless optical communication links by channel coding," Applied Optics, vol. 57, no. 9, pp. 2115-2120, Mar. 2018.

[39] T. Hamza and M. A. Khalighi, "On limitations of using silicon photomultipliers for underwater wireless optical communications," West Asian Colloquium on Optical Wireless Communications (WACOWC), Apr. 2019, accepted.

[40] F. Xu, M. A. Khalighi, P. Caussé, and S. Bourennane, "Channel coding and time-diversity for optical wireless links," Optics Express, vol. 7, no. 2, p. 872887 , Jan. 2009

[41] X. M. Zhu and J. M. Kahn, "Free-space optical communication through atomospheric turbulence channels," IEEE Transactions on Communications, vol. 50, no. 8, pp. 1293-1300, Aug. 2002. 